\title{
In vitro analysis of ovarian cancer response to cisplatin, carboplatin, and paclitaxel identifies common pathways that are also associated with overall patient survival
}

\author{
E Bicaku',2, Y Xiong ',2, DC Marchion',2, HS Chon', XB Stickles', N Chen', PL Judson ',3, A Hakam ${ }^{3,4}$, \\ J Gonzalez-Bosquet ${ }^{1,3}$, RM Wenham ${ }^{1,2,3}$, SM Apte ${ }^{1,3}$, W Fulp ${ }^{5}$, CL Cubitt ${ }^{6}$, D-T Chen and JM Lancaster $^{*, 1,2,3}$ \\ 'Department of Women's Oncology, H. Lee Moffitt Cancer Center and Research Institute, Tampa, FL 336 I2, USA; ${ }^{2}$ Experimental Therapeutics Program, \\ H. Lee Moffitt Cancer Center and Research Institute, Tampa, FL 336 I2, USA; ${ }^{3}$ Department of Oncologic Sciences, H. Lee Moffitt Cancer Center and \\ Research Institute, Tampa, FL 33612, USA; ${ }^{4}$ Department of Anatomic Pathology, H. Lee Moffitt Cancer Center and Research Institute, Tampa, \\ FL 336 12, USA; ${ }^{5}$ Biostatistics Core, H. Lee Moffitt Cancer Center and Research Institute, Tampa, FL 33612, USA; ${ }^{6}$ Translational Research Core, \\ H. Lee Moffitt Cancer Center and Research Institute, Tampa, FL 33612, USA
}

\begin{abstract}
BACKGROUND: Carboplatin and cisplatin, alone or in combination with paclitaxel, have similar efficacies against ovarian cancer (OVCA) yet exhibit different toxicity profiles. We characterised the common and unique cellular pathways that underlie OVCA response to these drugs and analyse whether they have a role in OVCA survival.

METHODS: Ovarian cancer cell lines $(n=36)$ were treated with carboplatin, cisplatin, paclitaxel, or carboplatin-paclitaxel (CPTX). For each cell line, $I_{50}$ levels were quantified and pre-treatment gene expression analyses were performed. Genes demonstrating expression $/ \mathrm{IC}_{50}$ correlations (measured by Pearson; $P<0.0 \mathrm{I}$ ) were subjected to biological pathway analysis. An independent $\mathrm{OVCA}$ clinico-genomic data set $(n=142)$ was evaluated for clinical features associated with represented pathways.

RESULTS: Cell line sensitivity to carboplatin, cisplatin, paclitaxel, and CPTX was associated with the expression of 77, 68, 64, and 25 biological pathways $(P<0.0 \mathrm{I})$, respectively. We found three common pathways when drug combinations were compared. Expression of one pathway ('Transcription/CREB pathway') was associated with OVCA overall survival.

CONCLUSION: The identification of the Transcription/CREB pathway (associated with OVCA cell line platinum sensitivity and overall survival) could improve patient stratification for treatment with current therapies and the rational selection of future OVCA therapy agents targeted to these pathways.

British Journal of Cancer (2012) 106, 1967-1975. doi:10.1038/bjc.2012.207 www.bjcancer.com
\end{abstract}

Published online 17 May 2012

(C) 2012 Cancer Research UK

Keywords: ovarian cancer; platinum agents; taxane; chemo-response; gene expression

The American Cancer Society ranks ovarian cancer (OVCA) as the fifth leading cause of cancer mortality in women, accounting for more deaths than any other gynaecologic cancer. In 2010 in the United States alone, approximately 22000 women had been predicted to be diagnosed with OVCA, with an estimated 14000 women dying of their disease (Jemal et al, 2010). This high mortality is attributed not only to the advanced stage (III/IV) at diagnosis observed for most patients (Herrin and Thigpen, 1999) but also to limited treatment options available for patients who develop acquired resistance to chemotherapy.

After cytoreductive surgery, most patients with advanced-stage disease receive systemic treatment with a platinum plus/minus taxane-based regimen. Clinical trials performed in the 1980 s and 1990s established the platinum compounds to be superior to alkylating agents, either alone or in combination with other nonplatinum drugs (Omura et al, 1986). Cisplatin and carboplatin do not differ significantly in their efficacy or effect on survival but

\footnotetext{
*Correspondence: Dr JM Lancaster;

E-mail: Johnathan.Lancaster@moffitt.org.

Received 3 January 2012; revised 12 April 2012; accepted 19 April 2012; published online 17 May 2012
}

do have markedly different side effects (Ozols et al, 2003). Paclitaxel, a compound extracted from Pacific yew tree bark, has been used as an antineoplastic agent since the 1980s and has been shown to improve OVCA progression-free and overall survival when combined with cisplatin or carboplatin in first-line therapy (McGuire et al, 1996). Randomised clinical trials comparing the combination of paclitaxel with either cisplatin or with carboplatin in patients with advanced-stage OVCA found no significant differences in efficacy between the two combination treatments, although there was generally an improved tolerability for the carboplatin-containing regimen (Neijt et al, 2000; du Bois et al, 2003; Ozols et al, 2003). Despite substantial clinical data demonstrating the superiority of platinum-based regimens $v \mathrm{~s}$ non-platinum regimens, the non-inferiority of carboplatin over cisplatin, and the benefit of paclitaxel combined with platinumbased regimens, the molecular basis to OVCA sensitivity to these drugs remains to be fully characterised.

The objectives of this study were to characterise the biological signalling pathways that underlie response to standard of care therapy and to further determine the influence of these pathways on overall survival from OVCA. To accomplish these objectives, we integrated chemo-sensitivity data from OVCA cell lines treated 
with cisplatin, carboplatin, and carboplatin plus paclitaxel with genome-wide expression data to provide insights into the unique and common signalling pathways that may comprise the biological basis of OVCA response to primary therapy agents. Although it is recognised that platinum-based agents induce cell death via initial mechanisms (and molecular pathways) that are distinct from those of the taxanes, it is also accepted that the two groups of drugs likely share a number of final biological and molecular pathways associated with the apoptotic process (Fisher, 1994; Eguchi et al, 1997; Zhan et al, 1999; Pestell et al, 2000; Wang et al, 2000), thus underlying the assumptions for this study. The identification of unique and shared determinants of drug response in OVCA has the potential to improve our understanding of OVCA chemo-response and also the stratification of patients for treatment with current therapies and the rational selection of agents targeted to these pathways for future treatment of OVCA.

\section{MATERIALS AND METHODS}

\section{Cell culture}

Ovarian cancer cell lines were obtained from the American Type Culture Collection (Manassas, VA, USA) (CAOV3, OV90, OVCAR3, SKOV3, TOV112D); from the European Collection of Cell Cultures, Salisbury, UK (A2780CP, A2780S); or from Kyoto University, Kyoto, Japan (CHI, CHIcisR, M41, M41CSR, Tyknu, and TyknuCisR) or were kind gifts from Dr Patricia Kruk, Department of Pathology, College of Medicine, University of South Florida, Tampa, FL, USA, and Susan Murphy, PhD, Department of OBGYN/Division of GYN Oncology, Duke University, Durham, NC, USA (A2008, C13, CAOV2, FUOV1, HeyA8, IGR-OV1, IMCC3, IMCC5, MCAS, OV2008, OVCA420, OVCA429, OVCA432, OVCA433, OVCAR4, OVCAR5, OVCAR8, Dov13, BG1, Ovary1847, OVCAR10, OVCAR2, SK-OV-4).

Cell lines were maintained in RPMI-1640 (Invitrogen, Carlsbad, CA, USA) supplemented with $10 \%$ fetal bovine serum (Fisher Scientific, Pittsburgh, PA, USA), 1\% sodium pyruvate, $1 \%$ penicillin/streptomycin (Cellgro, Manassas, VA, USA), and $1 \%$ nonessential amino acids (HyClone, Hudson, NH, USA). Mycoplasma testing was performed every 6 months following manufacturer's protocol (Lonza, Rockland, ME, USA).

\section{RNA extraction and microarray expression analysis}

RNA from 36 OVCA cell lines was extracted using RNeasy kit following manufacturer's recommendations (Qiagen, Valencia, CA, USA). Quality of the RNA was measured using an Agilent 2100 Bioanalyzer (Agilent Technologies, Santa Clara, CA, USA). The targets for Affymetrix DNA microarray analysis were prepared according to the manufacturer's instructions, and targets were hybridised to customised Human Affymetrix HuRSTA gene chips (HuRSTA-2a520709), which include 60607 probe sets and representation of 19308 genes (Gene Expression Omnibus accession number GSE34615).

\section{CellTiter-blue cell viability assays}

Drug activity was evaluated using a high-throughput CellTiter-Blue cell viability assay. Cells $\left(2.5 \times 10^{3}\right.$ per well $)$ were plated in 384 -well plates using complete media with $10 \%$ fetal bovine serum and allowed to adhere overnight. After cell adherence, increasing concentrations of cisplatin, carboplatin (Sigma-Aldrich, St Louis, MO, USA), paclitaxel (Sequoia Research Products Ltd., Pangbourne, UK), or carboplatin plus paclitaxel (carbotaxol: constant molar ratio of carboplatin to paclitaxel of $20000: 1$ ) were added to appropriate wells using an automated pipetting station. Four replicate wells were used for each drug concentration and vehicle controls. Drug dilutions initially consisted of 1.5 -fold serial dilutions from a maximum concentration of $100 \mu \mathrm{M}$. The cells were incubated with the drug for $72 \mathrm{~h}$ to ensure all cell lines underwent a minimum of two doublings. After drug incubation, $5 \mu \mathrm{l}$ of CellTiter-Blue reagent (Promega Corp., Madison, WI, USA) was added to each well. Fluorescence was read at $579 \mathrm{~nm}$ excitation/ $584 \mathrm{~nm}$ emission using a Synergy 4 microplate reader (Bio-Tek Instruments, Inc., Winooski, VT, USA). $\mathrm{IC}_{50}$ results were determined using a sigmoidal equilibrium model fit (XLfit 5.2, ID Business Solutions Ltd., Guildford, Surrey, UK). IC $_{50}$ was defined as the concentration of drug required for a $50 \%$ reduction in growth/viability.

\section{Statistical analysis}

Expression data from 36 OVCA cell lines were subjected to background correction and normalisation using the Robust Multichip Average algorithm in the Affymetrix Expression Console (http://www.affymetrix.com). Pearson correlation test was performed on individual gene expression values and $\mathrm{IC}_{50}$ results. Probe sets with $P<0.01$ were considered to have significant correlations with $\mathrm{IC}_{50}$ results and were uploaded to GeneGo MetaCore for pathway analysis (http://www.genego.com/metacore. php). Pathways with $P<0.05$ were considered significant, based on the GeneGo MetaCore statistical test for significance.

\section{Building signatures of pathway activity}

The principal component analysis methodology was used to derive a pathway gene expression signature with a corresponding 'pathway score' to represent an overall gene expression level for the pathways of interest. First, data were reduced into a small set of uncorrelated principal components. This set of principal components was generated based on its ability to account for variation. The first principal component analysis is used to represent the overall expression level for the pathway, as it accounts for the largest variability in the data. That is, pathway score is equal to $\sum w_{i} x_{i}$, a weighted average expression among the BAD pathway genes, where $x_{i}$ represents gene $i$ expression level, $\mathrm{w}_{i}$ is the corresponding weight (loading coefficient) with $\sum w_{i}^{2}=1$, and the $\mathrm{w}_{i}$ values maximise the variance of $\sum w_{i} x_{i}$.

\section{Validation of signatures in primary OVCA data sets}

The pathway gene expression signature scores developed in OVCA cell lines were evaluated in an independent clinico-genomic data set from 142 advanced-stage (III/IV) serous OVCA samples as previously described (Marchion et al, 2011). Gene expression data and the corresponding clinical parameters for these 142 OVCA samples are publicly available at www.ncbi.nlm.nih.gov/geo, accession number GSE23554. In brief, all 142 patients signed the IRB-approved, written informed consent forms, were known to have advanced-stage (III/IV) serous epithelial OVCA, and underwent primary cytoreductive surgery followed by primary therapy with a platinum-based regimen (with or without taxane or cyclophosphamide). Additional details are provided in the Supplementary Data section. Log-rank tests with Kaplan-Meier survival curves were used to test any association between the pathway scores ('high' $v s$ 'low' based on a median value cutoff) and overall survival for patients with OVCA. None of the data from the 142 OVCA samples was used to identify the principal component analysis signatures; the OVCA ovarian genomic data made up a completely independent evaluation set.

\section{CREB pathway analysis}

To further elucidate the biological basis to the association between CREB pathway expression and overall survival from OVCA, we performed a series of in silico analyses, including Pearson's correlation between growth doubling times for the NCI60 human 
cancer cell lines (and CREB pathway expression, quantified by CREB PC1 score). Affymetrix HG-U133A expression and doubling time data for the $60 \mathrm{NCI}$ cancer cell lines (6 leukaemia, 9 melanoma, 9 non-small cell lung, 7 colon, 6 central nervous system, 7 ovarian, 8 renal, 2 prostate, and 6 breast cancer cell lines) were obtained from NCI web sites (http://discover.nci.nih.gov/ cellminer/loadDownload.do and http://dtp.nci.nih.gov/docs/misc/ common_files/cell_list.html).

In light of the ability of the CREB pathway to influence transcription of other genes and molecular pathways, we evaluated differences in genome-wide expression in OVCAs that demonstrate high vs low CREB pathway expression. Using genomic data from 142 advanced-stage (III/IV) serous OVCAs (described above and previously (Marchion et al, 2011)), we studied genomic profiles from OVCAs that demonstrated a complete response (CR) to primary therapy for evidence of molecular signalling pathways that may differentially expressed in OVCAs that harbour high vs low expression levels of the CREB pathway (based on the median score threshold to define high $v s$ low). We performed a SAM $t$-test analysis to identify differentially expressed genes between ' $C R$ high-CREB' vs 'CR low-CREB' OVCAs. Differentially expressed genes $(\mathrm{FDR}=0)$ were subjected to Genego pathway analysis. In a preliminary analysis, using OVCA data from 23 patients for whom disease-free interval (DFI) information is available, we compared DFI for patients with low and high CREB PC1 scores (median score used as threshold).

\section{RESULTS}

The workflow for this study is summarised in Figure 1 . The $\mathrm{IC}_{50}$ values for 36 OVCA cell lines treated with increasing doses of

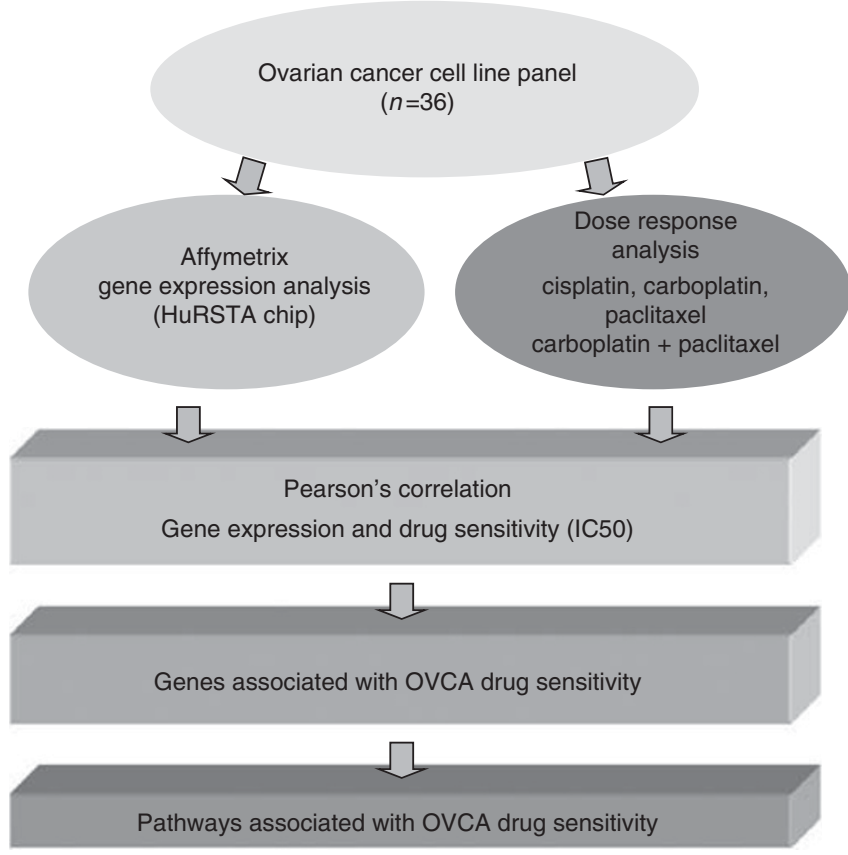

Figure I Experimental design flow chart. Gene expression analysis was performed on a panel of ovarian cancer cell lines $(n=36)$ using the custom Affymetrix HuRSTA chip. Each cell line was treated with increasing doses of carboplatin, cisplatin, paclitaxel, or carboplatin plus paclitaxel, and $I_{50}$ values were quantified using CellTiter-Blue cell viability assays. Pearson correlation coefficients were calculated for expression data and drug $\mathrm{IC}_{50}$ values. Genes associated with OVCA sensitivity (demonstrating expression/ $I C_{50}$ correlations, $P<0.0$ I) were subjected to GeneGo Metacore Pathway analysis. carboplatin, cisplatin, paclitaxel, and carboplatin plus paclitaxel treatment (Supplementary Table 1) were correlated with gene expression data for each drug (Pearson correlation coefficient, $P<0.01)$. Treatment of OVCA cell lines with carboplatin revealed 1201 genes whose differential expression correlated with carboplatin sensitivity $(P<0.01)$. This gene list corresponded to 77 biological pathways identified by GeneGo MetaCore analysis (Figure 2A). Cisplatin sensitivity correlated with the expression of 454 unique genes $(P<0.01)$, corresponding to 68 pathways. Twenty-three of these pathways were also correlated with carboplatin sensitivity (Figure 2A). Treatment with paclitaxel revealed 1025 genes associated with OVCA paclitaxel sensitivity $(P<0.01)$, representing 64 biological pathways (Figure 2B). Pearson correlation of the $\mathrm{IC}_{50}$ values and gene expression data for the OVCA cell lines treated with a combination of carboplatin and paclitaxel (20000:1 molar ratio) revealed 1049 differentially expressed genes $(P<0.01)$ and 25 pathways (Figure $2 \mathrm{~B}-\mathrm{D})$.

To distinguish between pathways that are unique to the mechanism of action of each drug and pathways that are common determinants of OVCA sensitivity, we identified pathways containing genes that were correlated with sensitivity to multiple drugs. Of the 25 pathways linked to sensitivity to carboplatin-paclitaxel combination therapy and the 68 pathways linked to cisplatin sensitivity, only 3 were identified in both analyses: 'Apoptosis and Survival/BAD Phosphorylation', 'Role of APC in Cell Cycle Regulation' (where APC is anaphase-promoting complex) and 'Transcription/CREB' (Figure 2D). Eight pathways were specifically correlated with carboplatin sensitivity, regardless of whether paclitaxel was used (Figure 2C). Two pathways were specifically correlated with sensitivity to paclitaxel treatment in both the presence and absence of carboplatin (Figure 2B).

To further distinguish core pathways involved in the response to chemotherapeutic agents in OVCA, we analysed the union of three individual comparisons: carboplatin sensitivity $v s$ cisplatin sensitivity, carboplatin-paclitaxel sensitivity $v s$ cisplatin sensitivity, and carboplatin-paclitaxel sensitivity $v s$ carboplatin sensitivity. We identified three pathways with a shared role in OVCA sensitivity to carboplatin and cisplatin single-agent treatment and carboplatin and paclitaxel combination treatment: 1) 'Apoptosis and Survival/ BAD Phosphorylation' 2) 'Role of APC in Cell Cycle Regulation,' and 3) 'Transcription/CREB' (Figure 3). Diagrams of the genes/ proteins involved in these pathways are provided in Figure 3 (GeneGo MetaCore output: September 29, 2010).

\section{Expression of the Transcription/CREB pathway is associated with OVCA clinical outcome}

Based on the above data, we utilised principal component analysis to develop expression signatures for both the Role of APC in Cell Cycle Regulation and the Transcription/CREB pathways. We have previously reported on an Apoptosis and Survival/BAD Phosphorylation pathway signature and its associations with chemosensitivity and overall survival (Marchion et al, 2011). A 55-gene APC in Cell Cycle Regulation pathway signature and a 103-gene Transcription/CREB pathway signature were generated and evaluated in an independent OVCA genomic data set (Supplementary Tables 2 and 3). Expression of the Transcription/CREB pathway gene signature was associated with overall survival from OVCA $(n=142, P \leqslant 0.0001$; Figure $4 \mathrm{~A})$. Furthermore, the OVCA genomic data set was evaluated with regard to Transcription/CREB pathway gene expression signature score and surgical cytoreductive (debulking, $n=141$, with debulking status unavailable for 1 of 142 patients) status (optimal: $<1 \mathrm{~cm}$; suboptimal: $>1 \mathrm{~cm}$ residual tumour at conclusion of surgery, $P<0.001$; Figure 4C) and also response to primary platinum-based therapy (complete or incomplete response, $P<0.0001$; Figure $4 \mathrm{~B}$ ). An association between low Transcription/CREB pathway gene expression signature score and favourable outcome was observed 
A

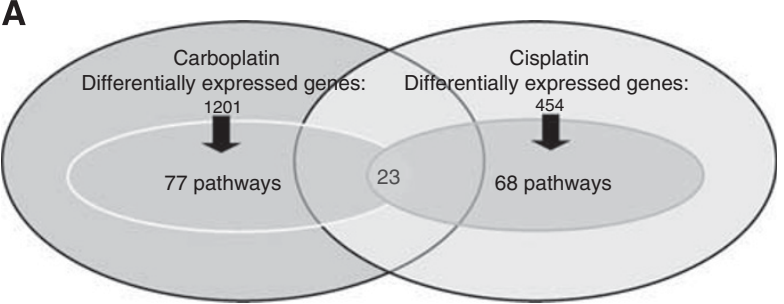

1. Apoptosis and survival/BAD phosphorylation

2. Apoptosis and survival/HTR1A signalling

3. Cell cycle/Role of APC in cell cycle regulation

4. Development/ACM2 and ACM4 activation of ERK

5. Development/Activation of Erk by ACM1, ACM3 and ACM5

6. Development/Activation of ERK by alpha-1 adrenergic receptors

7. Development/Activation of ERK by kappa-type opioid receptor

8. Development/Alpha-2 adrenergic receptor activation of ERK

9. Development/Angiotensin activation of ERK

10. Development/Angiotensin activation of ERK

11. Development/Beta-adrenergic receptors regulation of ERK

12. Development/G-proteins mediated regulation MARK-ERK signalling

13. Development/PDGF signalling via MAPK cascades

14. Development/Thyroliberin signalling

15. Immune response/HTR2A-induced activation of CPLA2

16. Immune response/MIF - the neuroendocrine-macrophage connector

17. Immune response/Neurotensin-induced activation of IL-8 in colonocytes

18. Membrane-bound ESR1: interaction with G-proteins signalling

19. Muscle contraction/Regulation of eNOS activity in cardiomyocytes

20. Neurophysiological process/Delta-type opioid receptor in the nervous system

21. Neurophysiological process/Melatonin signalling

22. Signal transduction/Erk interactions: inhibition of Erk

23. Transcription/CREB pathway

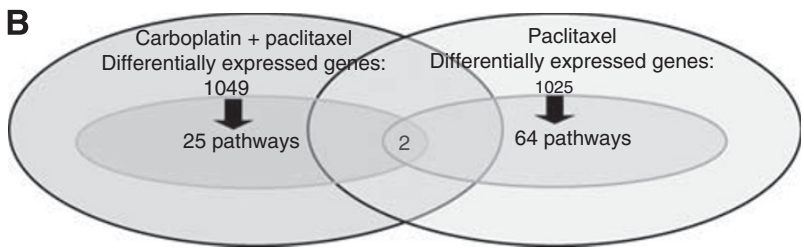

1. Cell cycle/Spindle assembly and chromosome separation

2. Transcription/Ligand-dependent transcription of retinoid-target genes

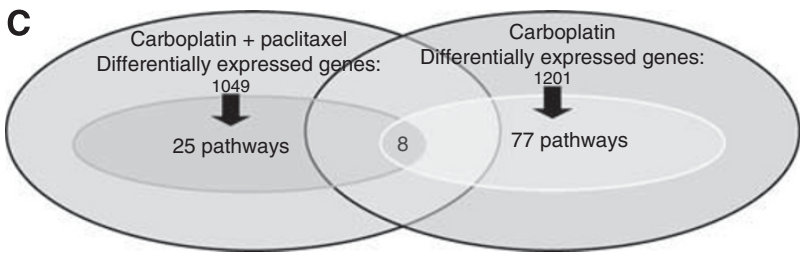

1. Apoptosis and survival/BAD phosphorylation

2. Cell cycle/ESR1 regulation of G1/S transition

3. Cell cycle/Regulation of G1/S transition (part 1)

4. Cell cycle/role of APC in cell cycle regulation

5. Cell cycle/Role of SCF complex in cell cycle regulation

6. Cell cycle/Spindle assembly and chromosome separation

7. Transcription/CREB pathway

8. Translation /Regulation of EIF2 activity

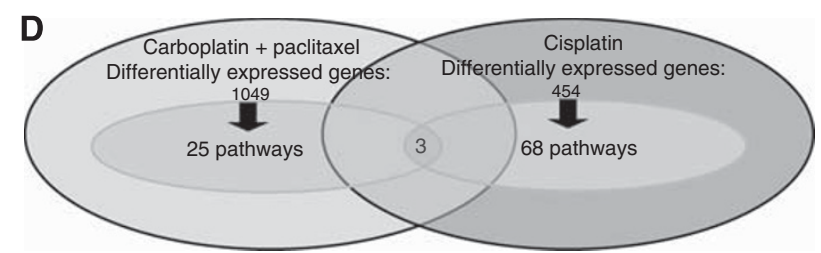

1. Apoptosis and survival/BAD phosphorylation cell cycle

2. Role of $A P C$ in cell cycle regulation

3. Transcription/CREB pathway

Figure 2 Shared biological pathway activation between chemotherapeutic agent treatments. The Pearson correlation test was performed to analyse the relationship between $I_{50}$ values and gene expression. Genes whose expression highly correlated with the $I C_{50}$ value associated with each drug $(P<0.0 \mathrm{I})$ were uploaded into the GeneGo MetaCore software to identify significantly represented pathways $(P<0.05)$. $(\mathbf{A})$ Ovarian cancer cell line sensitivity to carboplatin or cisplatin treatment was associated with 1201 and 454 genes, respectively. Genes associated with OVCA sensitivity to carboplatin and cisplatin were represented in 77 (carboplatin) and 68 (cisplatin) biological pathways. (B) Ovarian cancer cell line sensitivity to paclitaxel or carboplatin plus paclitaxel treatment was associated with the expression of 1025 and 1049 genes, respectively. Genes associated with OVCA sensitivity were represented in 64 (paclitaxel) and 25 (carboplatin plus paclitaxel) biological pathways. (C) Ovarian cancer cell line sensitivity to carboplatin plus paclitaxel treatment or carboplatin was associated with the expression of 1049 and 1201 genes, respectively. Genes associated with OVCA sensitivity were represented in 25 (carboplatin plus paclitaxel) and 77 (carboplatin) biological pathways. (D) Ovarian cancer cell line sensitivity to carboplatin plus paclitaxel treatment or cisplatin treatment was associated with the expression of 1049 and 454 genes, respectively. Genes associated with OVCA sensitivity were represented in 25 (carboplatin plus paclitaxel) and 68 (cisplatin) biological pathways.

in patients who underwent optimal and suboptimal debulking (optimal: adjusted $P=0.002$, suboptimal: adjusted $P=0.02$ ). Most importantly, OVCA patients with a low Transcription/CREB pathway gene expression signature score who underwent suboptimal debulking had survival data that trended toward superiority $v s$ patients with a high Transcription/CREB pathway gene expression signature score who underwent optimal debulking (adjusted $P=0.08$ ). Interestingly, patients who demonstrated a complete response to primary platinum-based therapy but had a low Transcription/CREB pathway gene expression signature score had notably superior survival than patients who demonstrated a complete response but had a high Transcription/CREB pathway gene expression signature score (adjusted $P=0.0001$ ). Patients who had an incomplete response to primary therapy had no difference in survival associated with their Transcription/ CREB pathway gene expression signature score $(P=0.27)$. When evaluated with debulking status and response to primary platinum-based therapy, grade, and age, the Cox proportional hazards multivariable model revealed that the Transcription/CREB pathway gene expression signature score was an independent variable associated with survival $(P=0.01)$.

The Role of APC in Cell Cycle Regulation pathway gene expression signature score demonstrated a trend toward an association with overall survival from OVCA, although results did not reach statistical significance $(n=142, P=0.1$, Figure $4 D)$. No association between the Role of APC in Cell Cycle Regulation pathway gene expression signature score and survival was observed within the group of patients who had optimal $(P=0.1384)$ or suboptimal $(P=0.6192)$ cytoreductive surgery or who had demonstrated a complete $(P=0.2154)$ or incomplete $(P=0.6984)$ response to primary platinum-based therapy.

\section{CREB pathway expression and OVCA biology}

We identified a correlation between expression of the CREB pathway (quantified by CREB PC1 score) and the doubling times of 60 human cancer cell lines (http://dtp.nci.nih.gov/docs/misc/ common_files/cell_list.html $)(P=0.006)$. As such, cancer cell lines 
A

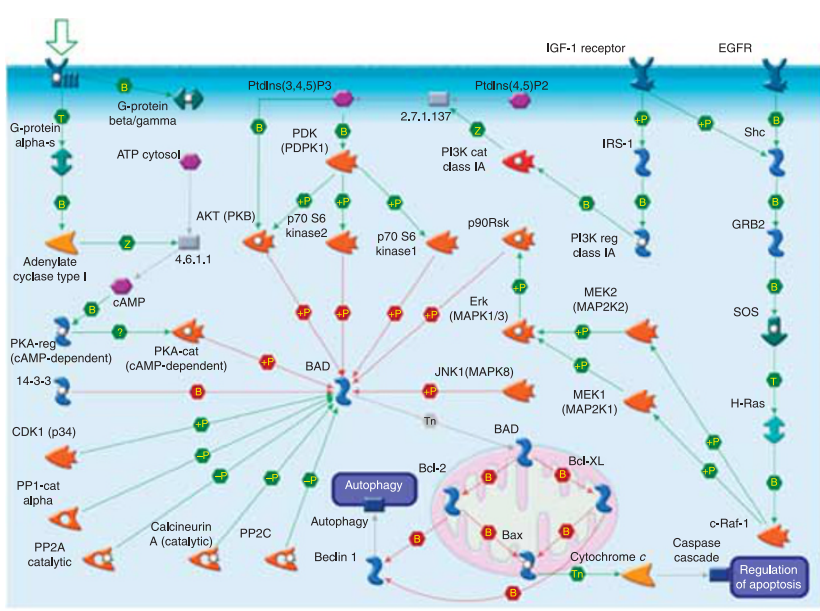

B

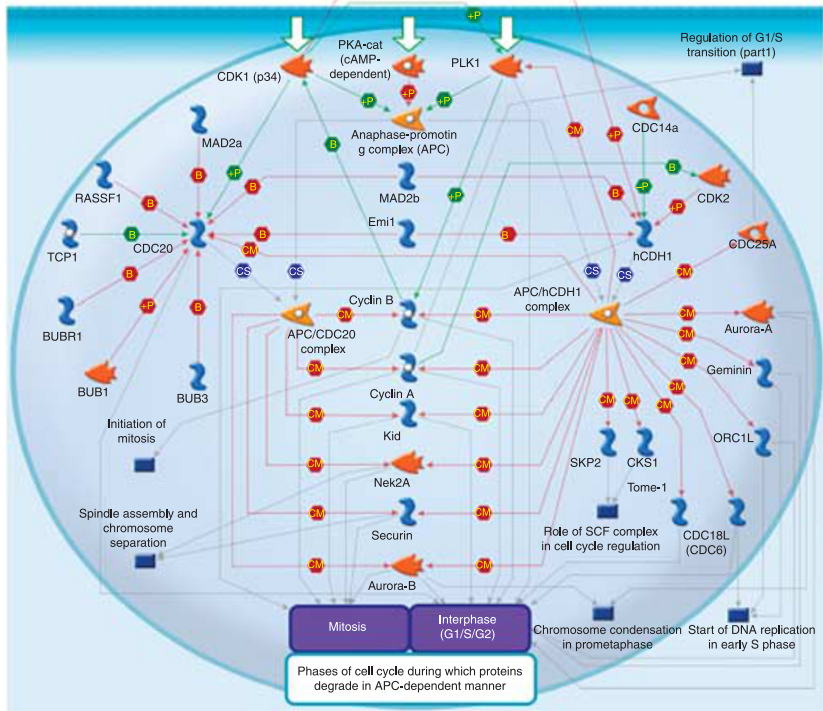

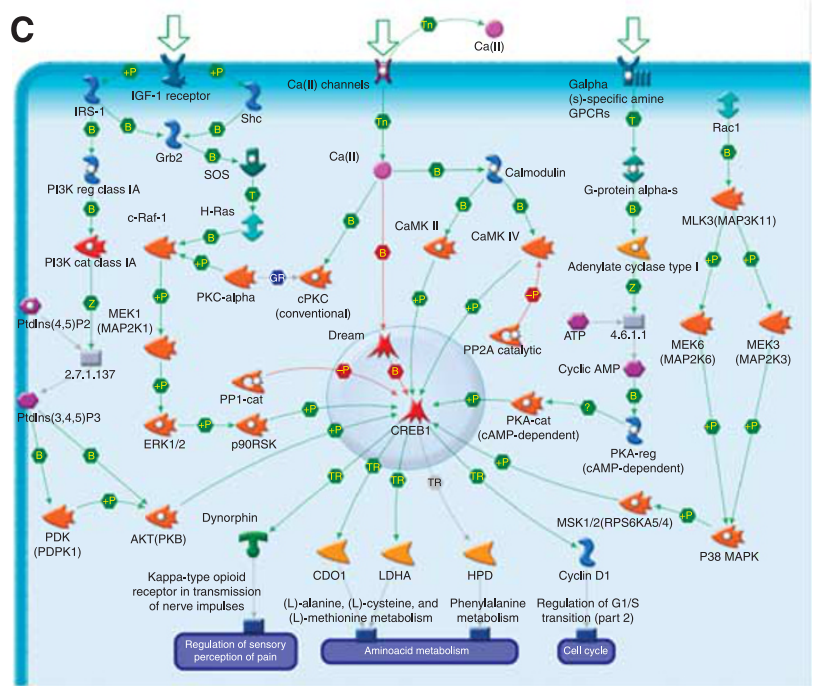

Figure 3 Common biological pathways activated in OVCA cells in response to chemotherapeutic agents. Three biological pathways are commonly activated in an OVCA cell line panel in multiple comparisons of chemotherapeutic agent response. (A) 'Apoptosis and Survival/BAD phosphorylation' pathway. (B) 'Cell Cycle/Role of APC in Cell Cycle Regulation' pathway. (C) 'Transcription/CREB' pathway.

with low doubling times (using median number as a threshold) demonstrated lower CREB pathway expression (PC1 scores) than cell lines with high doubling times $(P=0.003)$ (Figure 5). Furthermore, study of genomic data from patients who experienced a complete response to primary therapy revealed that OVCAs with high vs low CREB pathway expression also reflect differential expression of other pathways that have influence on tumour biology. SAM $t$-test identified differential expression of 4017 probe sets $(F D R=0)$ between 'CR low-CREB PC1' and 'CR highCREB PC1' score OVCAs. These differentially expressed genes included representation of 292 pathways $(P<0.05$; Supplementary Table S4), including cAMP signalling $\left(P=1.0 \times 10^{9}\right)$, CREB pathway $\left(P=1.0 \times 10^{6}\right)$ (both expected given CREB pathway function), IP3 signalling $\left(P=1.0 \times 10^{6}\right)$, apoptosis, survival, and BAD phosphorylation $\left(P=1.0 \times 10^{6}\right)$, G-protein-mediated regulation of MAPK-ERK signalling $\left(P=1.0 \times 10^{5}\right)$, and AKT signalling $\left(P=1.0 \times 10^{5}\right)$. Finally, although the findings did not reach statistical significance, and the number of patients for whom DFI is available is low $(n=23)$, we did observe a trend toward shorter DFI in patients with OVCAs harbouring high CREB scores $(P=0.6$, data not shown).

\section{DISCUSSION}

Cancer cells employ a variety of strategies to deregulate apoptosis, cell cycle progression, and cellular metabolism during the course of their evolution (Hanahan and Weinberg, 2011). In this study, integration of OVCA cell line chemo-sensitivity and genome-wide expression data implicated pathways, which include BAD, APC, and CREB, as key mediators of sensitivity to chemotherapeutic agents, that, when deregulated, may contribute to the acquired drug resistance frequently observed in OVCA patients. Furthermore, when we integrated genome-wide expression results with clinical outcomes data from patients with advanced-stage (III/IV) serous OVCA, we found that these pathways are not only associated with in vitro drug sensitivity but also with patient survival. It is perhaps not surprising that there are both common and unique pathways that underlie OVCA platinum plus/minus taxane response; those that are associated with the differing initial mechanisms that ultimately lead to apoptosis, and those common (and potentially unique) pathways that may be associated with the apoptotic process. Paclitaxel is a microtubule-stabilising agent with potent anti-proliferative and anti-migratory activity for 

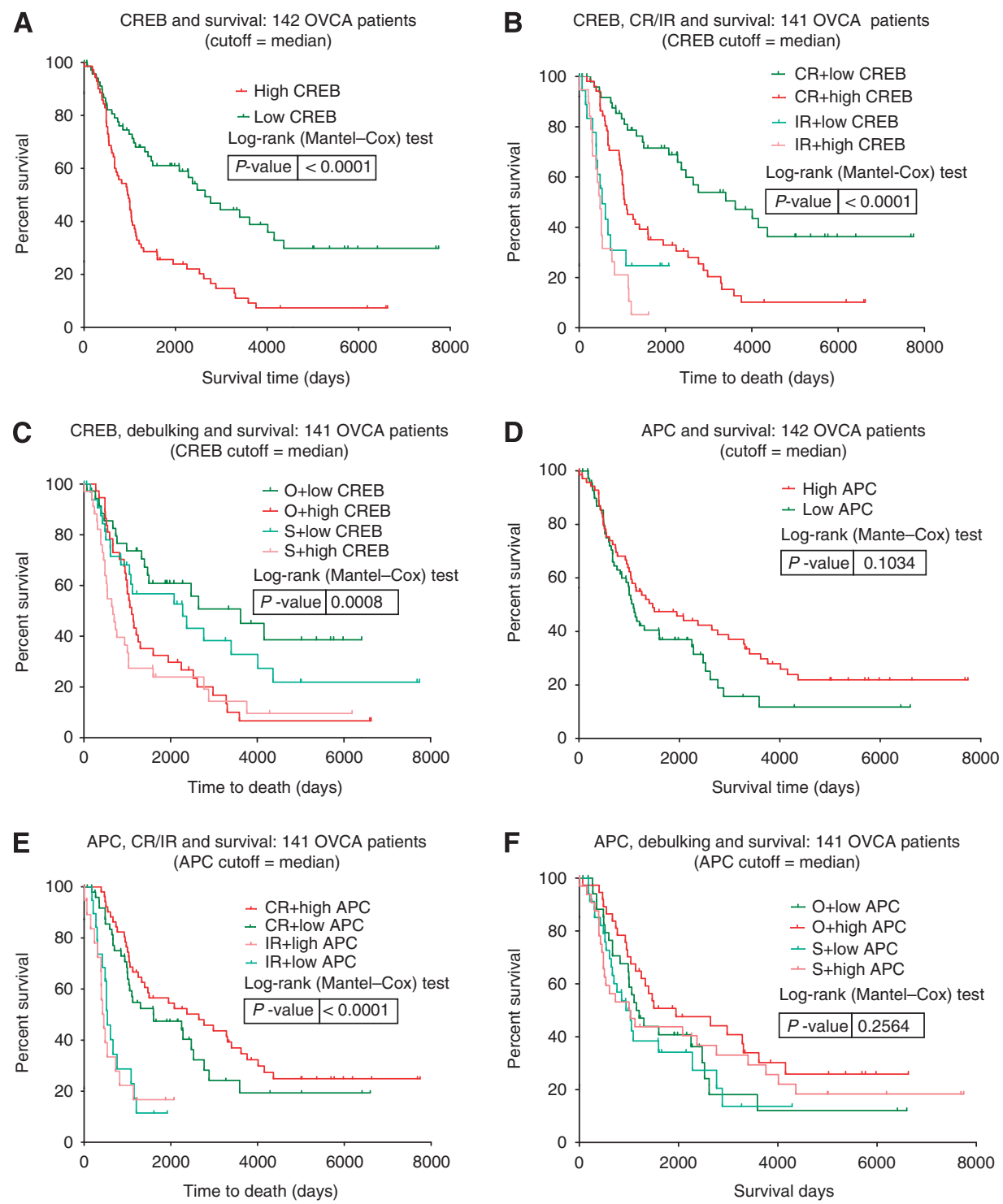

Figure 4 Low CREB pathway and High APC pathway signature principal component analysis (PCA) scores are associated with favourable clinical outcome. Kaplan-Meier curves depict the association between CREB-pathway signature PCA score $(\mathbf{A}-\mathbf{C})$ and APC-pathway signature PCA score (D-F) and overall survival from cancer for ONCA data sets. Information available for 14I of I42 samples. Log-rank test P-values indicate significance. Abbreviations: $C R=$ complete response; $I R=$ incomplete response; $O=$ optimal; $S=$ suboptimal.

multiple cell types, including fibroblasts, epithelial cells and tumour cells (Thyberg, 1984; Hruban et al, 1989; Bhalla et al, 1993; Donaldson et al, 1994; Rowinsky and Donehower, 1995). It has been established that paclitaxel kills cancer cells via microtubule binding, induction of kinetic suppression of microtubule dynamics, and cell cycle arrest in mitosis, that leads to induced apoptosis via activation of cyclin-dependent kinases, and the c-Jun $\mathrm{N}$-terminal kinase/stress-activated protein kinase (JNK/SAPK) (Wang et al, 2000). In contrast, the primary mechanism of cisplatin-induced cytotoxicity is generally accepted to be associated with DNA damage that subsequently leads to apoptosis (Fisher, 1994), though apoptosis may also be caused by damage to cytoplasmic or nuclear proteins including molecules involved in cellular energy (i.e., ATP) or proteins directly or indirectly involved in the apoptotic process (i.e., p53, Bax, Bcl, and caspases), leading to necrotic cell death (Perez, 1998). In fact, both apoptosis and necrosis have been observed in cisplatin-treated cells (Eguchi et al, 1997; Zhan et al, 1999; Pestell et al, 2000). In light of the different primary mechanisms of action (and underlying molecular signalling pathways) of platinum-based agents $v s$ the microtubule-binding taxanes, there also appear to be potential convergences of action via common pathways that lead to-and are associated with-the apoptotic processes. Future studies of both the common and unique pathways associated with OVCA platinum- plus/minus taxane response may also shed light on the relative contributions of identified unique and common pathways to differing mechanisms of cell death.

Our primary finding was regarding the Transcription/CREB pathway. Expression of this pathway was significantly associated with survival in patients who demonstrate a complete response to primary platinum-based therapy (adjusted $P=0.0001$ ), suggesting that expression of this pathway not only influences OVCA response to platinum-based therapy but also to the behaviour of de novo platinum-sensitive tumours subsequent to a complete response to primary therapy such that survival is impacted. We observed no such effect in patients who experience an incomplete response to platinum, suggesting that, although expression of the pathway is associated with primary chemo-sensitivity, it has no influence on the post-treatment phenotypic behaviour of OVCAs that are resistant to platinum. Notably, the influence of the 
CREB PC1: low vs high doubling time

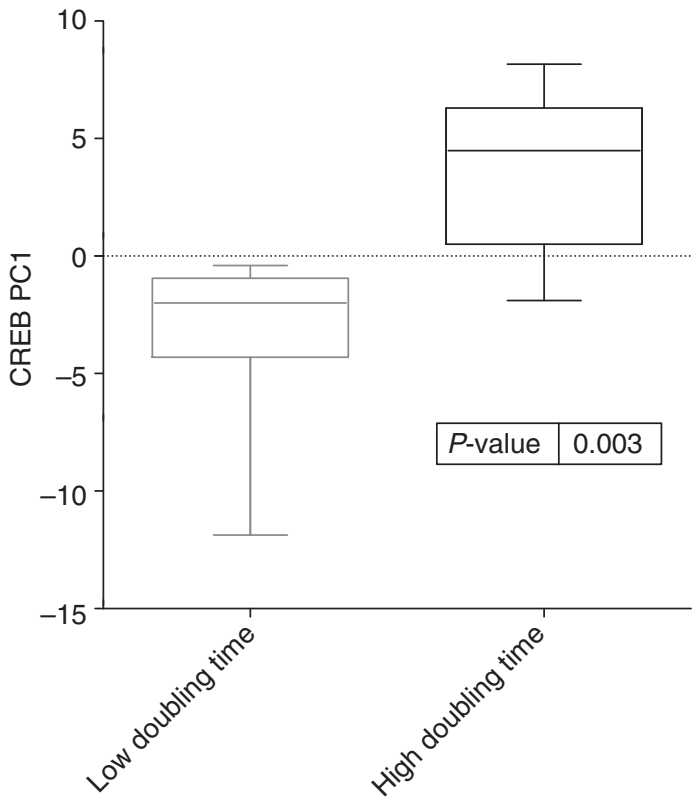

Figure 5 Expression of the CREB pathway is associated with the growth rates of cancer cells. Box plot depicting the association between CREB pathway principal component analysis mRNA expression score and cell line doubling times of the $\mathrm{NCl} 60$ cancer cell line panel. Median doubling time was used as a cutoff. Boxes indicate $95 \%$ confidence.

Transcription/CREB pathway on overall survival also approached statistical significance, despite cytoreductive status (the volume of residual disease at the completion of primary surgery), which is considered one of the most important clinical determinants of survival for patients with OVCA. Our analyses demonstrated a statistically significant correlation between CREB pathway expression and the doubling times of 60 human cancer cell lines, further supporting the view that the influence of the CREB pathway on overall survival from OVCA may be due to an effect on biological processes over-and-above chemo-sensitivity. Indeed, when we explored genes and pathways that are differentially expressed in OVCAs that demonstrated complete responses to primary therapy but that harboured high $v s$ low CREB pathway scores, we identified a series of molecular signalling pathways known to influence tumour behaviour, cellular proliferation and apoptosis (e.g., AKT, G-protein signalling, MAP/ERK, BAD). Our findings suggest that CREB pathway activity influence OVCA cell sensitivity to chemotherapeutic agents, but also, perhaps independently, influence cellular proliferation, which can also influence chemosensitivity. It is possible, therefore, that expression of the pathway influences clinical outcome from OVCA via direct effects on chemo-sensitivity, but also indirectly via transcriptional influences on additional pathways that impact cellular proliferation and apoptosis, even following a complete response to primary treatment. These findings highlight the importance of the CREB pathway in many aspects of OVCA phenotypic behaviour and may have substantial implications for the future direction of research into disease development, response to therapy, and clinical outcome.

cAMP response element-binding protein (CREB) is a transcription factor that can be activated by multiple pathways that affect cellular cAMP levels, and its many targets have important roles in cellular metabolism, proliferation, and survival (Mayr and Montminy, 2001; Zhang et al, 2005). G-protein-coupled receptors, transmembrane proteins that transduce extracellular signals to intracellular effector pathways via heterotrimeric $G$ proteins, activate the enzyme adenylate cyclase, which catalyses the conversion of ATP to CAMP. The cAMP then binds the regulatory subunits of protein kinase A (PKA), which leads to the release of the PKA catalytic subunits (Lappano and Maggiolini, 2011). Active PKA migrates to the nucleus, where it phosphorylates CREB (Gonzalez and Montminy, 1989). Phosphorylated CREB then binds its coactivator $\mathrm{CBP} / \mathrm{p} 300$, a histone acetyltransferase, and together $\mathrm{CREB}$ and $\mathrm{CBP} / \mathrm{p} 300$ activate the transcription of genes whose promoters contain a CRE (cAMP response element) sequence (Bannister and Kouzarides, 1996; Parker et al, 1996). Overexpression of CREB may be required for the proliferation and survival of human cancers (Bonni et al, 1999; Siu and Jin, 2007). Additionally, the CREB coactivator $\mathrm{CBP} / \mathrm{p} 300$ is mutated in several human cancers, with loss of $\mathrm{CBP} / \mathrm{p} 300$ histone acetyltransferase activity potentially impacting the expression of several tumour suppressor targets (Iyer et al, 2004). Clearly, additional functional analyses are warranted to better define the mechanistic basis to these observations.

BCL-xL/BCL-2-associated death promoter (BAD) was first identified in a yeast two-hybrid screen for BCL-2 interactors (Yang et al, 1995). Heterodimerisation between BAD and BCL-2, $\mathrm{BCL}-\mathrm{xL}$, or BCL-W promotes apoptosis through the displacement of BAK and BAX (Yang et al, 1995; Holmgreen et al, 1999). The interaction between $\mathrm{BAD}$ and its partners is dependent on the BAD BH3 domain (Zha et al, 1997). Upon apoptotic stimuli, BAD translocates from the cytosol to the mitochondria (Zha et al, 1996; Jia et al, 1999), where it neutralises the anti-apoptotic proteins and promotes mitochondrial membrane permeabilisation (Datta et al, 2000; Roy et al, 2009). Subversion of BAD-mediated apoptosis may thus be an important mechanism by which tumour cells acquire resistance to apoptotic stimuli. A role of BAD-induced apoptosis in tumour suppression is supported by the identification of BAD mutations in colon cancer that disrupt binding to BCL-2 and $\mathrm{BCL}-\mathrm{xL}$ and the observation that BAD-deficient mice are prone to diffuse B-cell lymphoma (Ranger et al, 2003; Lee et al, 2004). Furthermore, because post-translational modification of BAD represents a key control point between cell survival and apoptosis, $\mathrm{BAD}$ phosphorylation is frequently deregulated in cancer. Activation of multiple signalling pathways by growth factors, cytokines, or oncogenic mutations can contribute to increased BAD phosphorylation, with aberrant kinase and phosphatase activity leading to $\mathrm{BAD}$ inactivation and resistance to cell death signalling in tumours (Danial, 2008).

The anaphase-promoting complex/cyclosome (APC/C) is a multi-subunit ubiquitin ligase required for regulation of cell cycle progression and mitotic exit. The APC/C consists of multiple core subunits and an activator component. The APC/C is tightly regulated during the cell cycle via the cyclical binding of the Cdc20- (cell division cycle 20) and Cdh1- (cell division cycle homologue 1) activating subunits. The major role of APC/C is to target substrates for proteasomal degradation in an orchestrated manner, with $\mathrm{Cdc} 20$ and $\mathrm{Cdh} 1$ mediating substrate recognition to ensure that mitotic entry and exit occur correctly. The APC/C $\mathrm{C}^{\mathrm{Cdc} 20}$ is active from anaphase to the end of mitosis, and $A P C / C^{C d h} 1$ is active from late mitosis to the $\mathrm{G}_{1}-\mathrm{S}$ transition. The $\mathrm{APC} / \mathrm{C}^{\mathrm{Cdc} 20}$ mediated destruction of targets such as securin and cyclin B drives chromosome separation and exit from mitosis. As mitotic cyclins are degraded, cyclin-dependent kinase activity decreases. By late $\mathrm{M}$ phase, Cdh1 is no longer phosphorylated and can bind to the core APC/C subunits. The APC/C ${ }^{\mathrm{Cdh} 1}$ has multiple targets, including Cdc20, mitotic cyclins, mitotic kinases, and inhibitors of DNA replication origin licensing. Collectively, degradation of APC/C $\mathrm{C}^{\mathrm{Cdh} 1}$ targets ensures that cells do not duplicate their DNA and/or enter mitosis prematurely (Nakayama and Nakayama, 2006). Because of the critical role of $\mathrm{APC} / \mathrm{C}$ in regulating chromosome segregation during mitosis, deregulation of the complex could cause genomic instability and contribute to tumourigenesis. Indeed, loss-offunction mutations of core APC/C subunits has been observed in 
colorectal cancer cells, upregulation of Cdc20 has been observed in several human cancers, and loss of Cdh1 causes chromosomal instability and tumour formation in mice (Wang et al, 2003; Nakayama and Nakayama, 2006; Garcia-Higuera et al, 2008).

The $\mathrm{BAD}, \mathrm{CREB}$, and $\mathrm{APC} / \mathrm{C}$ may be linked by common signalling molecules, as signalling pathways converge in many ways to regulate cellular proliferation and survival at both the transcriptional and post-transcriptional levels. For example, PKA phosphorylation activates CREB and inactivates BAD and APC/

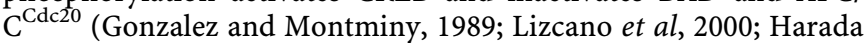
et al, 2001; Searle et al, 2004). AKT also phosphorylates BAD and $\mathrm{CREB}$, and $\mathrm{AKT}$ phosphorylation protects some $\mathrm{APC} / \mathrm{C}^{\mathrm{Cdh} 1}$ substrates from degradation (Datta et al, 1997; del Peso et al, 1997; Gao et al, 2009). PTEN can also promote the association between APC/C and Cdh1 to prevent S phase entry (Song et al, 2011). Cyclin-dependent kinases phosphorylate BAD, APC/C, and CBP/p300 (Ait-Si-Ali et al, 1998; Zachariae et al, 1998; Konishi et al, 2002). There may also be more direct crosstalk between BAD, CREB, and APC. BCL-xL/BCL-2-associated death promoter (BAD) has been linked to regulation of cell cycle progression and has been found at CREs in cyclin genes (Chattopadhyay et al, 2001; Fernando et al, 2007). The APC/C subunits APC5 and APC7 directly bind $\mathrm{CBP} / \mathrm{p} 300$, stimulate $\mathrm{CBP} / \mathrm{p} 300$ histone acetyltransferase activity, and potentiate $\mathrm{CBP} / \mathrm{p} 300$-dependent transcription (Turnell et al, 2005). cAMP response element-binding protein may also directly impact cell survival by binding to CREs in the promoters of anti-apoptotic factor genes such as BCL-2 to induce their transcription (Wilson et al, 1996).

Based on the known interactions between these proteins, BAD, $\mathrm{APC} / \mathrm{C}$, and $\mathrm{CREB}$ may potentially interact in a common pathway to induce cell cycle arrest and death in response to mitotic or metabolic stress caused by carboplatin, cisplatin, and paclitaxel. Deregulation of components of these pathways may thus lead to acquired drug resistance. Indeed, abnormal cell cycle progression, defective apoptosis, and metabolic reprogramming have been implicated as key mechanisms of OVCA chemoresistance

\section{REFERENCES}

Ait-Si-Ali S, Ramirez S, Barre FX, Dkhissi F, Magnaghi-Jaulin L, Girault JA, Robin P, Knibiehler M, Pritchard LL, Ducommun B, Trouche D, Harel-Bellan A (1998) Histone acetyltransferase activity of CBP is controlled by cycle-dependent kinases and oncoprotein E1A. Nature 396(6707): 184-186

Bannister AJ, Kouzarides T (1996) The CBP co-activator is a histone acetyltransferase. Nature 384(6610): 641-643

Bhalla K, Ibrado AM, Tourkina E, Tang C, Mahoney ME, Huang Y (1993) Taxol induces internucleosomal DNA fragmentation associated with programmed cell death in human myeloid leukemia cells. Leukemia 7(4): 563-568

Bonni A, Brunet A, West AE, Datta SR, Takasu MA, Greenberg ME (1999) Cell survival promoted by the Ras-MAPK signaling pathway by transcription-dependent and -independent mechanisms. Science 286(5443): 1358-1362

Chattopadhyay A, Chiang CW, Yang E (2001) BAD/BCL-[X(L)] heterodimerization leads to bypass of G0/G1 arrest. Oncogene 20(33): 4507-4518

Danial NN (2008) BAD: undertaker by night, candyman by day. Oncogene 27(Suppl 1): S53-S70

Datta SR, Dudek H, Tao X, Masters S, Fu H, Gotoh Y, Greenberg ME (1997) Akt phosphorylation of BAD couples survival signals to the cell-intrinsic death machinery. Cell 91(2): 231-241

Datta SR, Katsov A, Hu L, Petros A, Fesik SW, Yaffe MB, Greenberg ME (2000) 14-3-3 proteins and survival kinases cooperate to inactivate BAD by BH3 domain phosphorylation. Mol Cell 6(1): 41-51

del Peso L, Gonzalez-Garcia M, Page C, Herrera R, Nunez G (1997) Interleukin-3-induced phosphorylation of $\mathrm{BAD}$ through the protein kinase Akt. Science 278(5338): 687-689

Donaldson KL, Goolsby GL, Kiener PA, Wahl AF (1994) Activation of p34cdc2 coincident with taxol-induced apoptosis. Cell Growth Differ 5(10): 1041-1050
(Takahashi et al, 2005; Hajra et al, 2008; Etemadmoghadam et al, 2009; Montopoli et al, 2011).

We have shown that integration of chemo-sensitivity and genome-wide expression data can provide a more comprehensive understanding of the mechanisms involved in drug responses. Our results demonstrate that characterisation of gene expression signatures in OVCA patients may be useful for characterisng responses to treatment and also in developing an understanding of the biology that drives clinical outcome and survival. Furthermore, our identification of $\mathrm{BAD}, \mathrm{APC} / \mathrm{C}$, and $\mathrm{CREB}$ as key players in the cytotoxicity of carboplatin, cisplatin, and paclitaxel in OVCA cell lines suggests that targeted therapies against regulators of these proteins may be worthy of study in chemoresistant OVCAs.

\section{ACKNOWLEDGEMENTS}

We thank Rasa Hamilton (Moffitt Cancer Centre) for editorial assistance. We also acknowledge Carolyn Buser-Doepner and Merck Pharmaceuticals for their contributions. This project was supported in part by National Cancer Institute Grant R21 CA-110499-01A2, the Ocala Royal Dames For Cancer Research Inc., the Phi Beta Psi Sorority, the Hearing the Ovarian Cancer Whisper, Jacquie Liggett Foundation, the Ovarian Cancer Research Fund, and the US Army Medical Research and Materiel Command under Award No. DAMD17-02-2-0051.

\section{Disclaimer}

Opinions, interpretations, conclusions and recommendations are those of the author and are not necessarily endorsed by the funding agencies.

Supplementary Information accompanies the paper on British Journal of Cancer website (http://www.nature.com/bjc) du Bois A, Lūck H-J, Meier W, Adams H-P, Mōbus V, Costa S, Bauknecht T, Richter B, Warm M, Schrōder W, Olbricht S, Nitz U, Jackisch C, Gn Emons, Wagner U, Kuhn W, Pfisterer J, Group FtAGkOOCS (2003) A randomized clinical trial of cisplatin/paclitaxel versus carboplatin/ paclitaxel as first-line treatment of ovarian cancer. $J$ Natl Cancer Inst 95(17): 1320-1329

Eguchi Y, Shimizu S, Tsujimoto Y (1997) Intracellular ATP levels determine cell death fate by apoptosis or necrosis. Cancer Res 57(10): 1835-1840

Etemadmoghadam D, deFazio A, Beroukhim R, Mermel C, George J, Getz G, Tothill R, Okamoto A, Raeder MB, Harnett P, Lade S, Akslen LA, Tinker AV, Locandro B, Alsop K, Chiew YE, Traficante N, Fereday S, Johnson D, Fox S, Sellers W, Urashima M, Salvesen HB, Meyerson M, Bowtell D (2009) Integrated genome-wide DNA copy number and expression analysis identifies distinct mechanisms of primary chemoresistance in ovarian carcinomas. Clin Cancer Res 15(4): 1417-1427

Fernando R, Foster JS, Bible A, Strom A, Pestell RG, Rao M, Saxton A, Baek SJ, Yamaguchi K, Donnell R, Cekanova M, Wimalasena J (2007) Breas cancer cell proliferation is inhibited by BAD: regulation of cyclin D1. J Biol Chem 282(39): 28864-28873

Fisher DE (1994) Apoptosis in cancer therapy: crossing the threshold. Cell 78(4): 539-542

Gao D, Inuzuka H, Tseng A, Chin RY, Toker A, Wei W (2009) Phosphorylation by Akt1 promotes cytoplasmic localization of Skp2 and impairs APCCdh1-mediated Skp2 destruction. Nat Cell Biol 11(4): $397-408$

Garcia-Higuera I, Manchado E, Dubus P, Canamero M, Mendez J, Moreno S, Malumbres M (2008) Genomic stability and tumour suppression by the APC/C cofactor Cdh1. Nat Cell Biol 10(7): 802-811

Gonzalez GA, Montminy MR (1989) Cyclic AMP stimulates somatostatin gene transcription by phosphorylation of CREB at serine 133. Cell 59(4): $675-680$ 
Hajra KM, Tan L, Liu JR (2008) Defective apoptosis underlies chemoresistance in ovarian cancer. Adv Exp Med Biol 622: 197-208

Hanahan D, Weinberg RA. Hallmarks of cancer: the next generation. Cell (2011) 144(5): 646-674

Harada H, Andersen JS, Mann M, Terada N, Korsmeyer SJ (2001) p70S6 kinase signals cell survival as well as growth, inactivating the proapoptotic molecule BAD. Proc Natl Acad Sci USA 98(17): 9666-9670

Herrin VE, Thigpen JT (1999) Chemotherapy for ovarian cancer: current concepts. Semin Surg Oncol 17(3): 181-188

Holmgreen SP, Huang DC, Adams JM, Cory S (1999) Survival activity of Bcl-2 homologs Bcl-w and Al only partially correlates with their ability to bind pro-apoptotic family members. Cell Death Differ 6(6): 525-532

Hruban RH, Yardley JH, Donehower RC, Boitnott JK (1989) Taxol toxicity. Epithelial necrosis in the gastrointestinal tract associated with polymerized microtubule accumulation and mitotic arrest. Cancer 63(10): 1944-1950

Iyer NG, Ozdag H, Caldas C (2004) p300/CBP and cancer. Oncogene 23(24): 4225-4231

Jemal A, Siegel R, Xu J, Ward E. Cancer statistics (2010) CA Cancer J Clin 60(5): 277-300

Jia L, Macey MG, Yin Y, Newland AC, Kelsey SM (1999) Subcellular distribution and redistribution of Bcl-2 family proteins in human leukemia cells undergoing apoptosis. Blood 93(7): 2353-2359

Konishi Y, Lehtinen M, Donovan N, Bonni A (2002) Cdc2 phosphorylation of BAD links the cell cycle to the cell death machinery. Mol Cell 9(5): 1005-1016

Lappano R, Maggiolini M. G protein-coupled receptors: novel targets for drug discovery in cancer. Nat Rev Drug Discov (2011) 10(1): 47-60

Lee JW, Soung YH, Kim SY, Nam SW, Kim CJ, Cho YG, Lee JH, Kim HS, Park WS, Kim SH, Lee JY, Yoo NJ, Lee SH (2004) Inactivating mutations of proapoptotic Bad gene in human colon cancers. Carcinogenesis 25(8): 1371-1376

Lizcano JM, Morrice N, Cohen P (2000) Regulation of BAD by cAMP-dependent protein kinase is mediated via phosphorylation of a novel site, Ser155. Biochem J 349(Pt 2): 547-557

Marchion DC, Cottrill HM, Xiong Y, Chen N, Bicaku E, Fulp WJ, Bansal N, Chon HS, Stickles XB, Kamath SG, Hakam A, Li L, Su D, Moreno C, Judson PL, Berchuck A, Wenham RM, Apte SM, Gonzalez-Bosquet J, Bloom GC, Eschrich SA, Sebti S, Chen DT, Lancaster JM (2011) BAD phosphorylation determines ovarian cancer chemosensitivity and patient survival. Clin Cancer Res 17(19): 6356-6366

Mayr B, Montminy M (2001) Transcriptional regulation by the phosphorylation-dependent factor CREB. Nat Rev Mol Cell Biol 2(8): 599-609

McGuire WP, Hoskins WJ, Brady MF, Kucera PR, Partridge EE, Look KY, Clarke-Pearson DL, Davidson M (1996) Cyclophosphamide and cisplatin compared with paclitaxel and cisplatin in patients with stage III and stage IV ovarian cancer. $N$ Engl J Med 334(1): 1-6

Montopoli M, Bellanda M, Lonardoni F, Ragazzi E, Dorigo P, Froldi G, Mammi S, Caparrotta L (2011) 'Metabolic reprogramming' in ovarian cancer cells resistant to cisplatin. Curr Cancer Drug Targets 11(2): 226-235

Nakayama KI, Nakayama K (2006) Ubiquitin ligases: cell-cycle control and cancer. Nat Rev Cancer 6(5): 369-381

Neijt JP, Engelholm SA, Tuxen MK, Sorensen PG, Hansen M, Sessa C, de Swart CA, Hirsch FR, Lund B, van Houwelingen HC (2000) Exploratory phase III study of paclitaxel and cisplatin versus paclitaxel and carboplatin in advanced ovarian cancer. J Clin Oncol 18(17): 3084-3092

Omura G, Blessing JA, Ehrlich CE, Miller A, Yordan E, Creasman WT, Homesley HD (1986) A randomized trial of cyclophosphamide and doxorubicin with or without cisplatin in advanced ovarian carcinoma. A gynecologic oncology group study. Cancer 57(9): 1725-1730

Ozols RF, Bundy BN, Greer BE, Fowler JM, Clarke-Pearson D, Burger RA, Mannel RS, DeGeest K, Hartenbach EM, Baergen R (2003) Phase III trial of carboplatin and paclitaxel compared with cisplatin and paclitaxel in patients with optimally resected stage III ovarian cancer: a gynecologic oncology group study. J Clin Oncol 21(17): 3194-3200
Parker D, Ferreri K, Nakajima T, LaMorte VJ, Evans R, Koerber SC, Hoeger C, Montminy MR (1996) Phosphorylation of CREB at Ser-133 induces complex formation with CREB-binding protein via a direct mechanism. Mol Cell Biol 16(2): 694-703

Perez RP (1998) Cellular and molecular determinants of cisplatin resistance. Eur J Cancer 34(10): 1535-1542

Pestell KE, Hobbs SM, Titley JC, Kelland LR, Walton MI (2000) Effect of p53 status on sensitivity to platinum complexes in a human ovarian cancer cell line. Mol Pharmacol 57(3): 503-511

Ranger AM, Zha J, Harada H, Datta SR, Danial NN, Gilmore AP, Kutok JL, Le Beau MM, Greenberg ME, Korsmeyer SJ (2003) Bad-deficient mice develop diffuse large B cell lymphoma. Proc Natl Acad Sci USA 100(16): 9324-9329

Rowinsky EK, Donehower RC (1995) Paclitaxel (taxol). N Engl J Med 332(15): 1004-1014

Roy SS, Madesh M, Davies E, Antonsson B, Danial N, Hajnoczky G (2009) Bad targets the permeability transition pore independent of Bax or Bak to switch between $\mathrm{Ca} 2+$-dependent cell survival and death. Mol Cell 33(3): 377-388

Searle JS, Schollaert KL, Wilkins BJ, Sanchez Y (2004) The DNA damage checkpoint and PKA pathways converge on APC substrates and Cdc20 to regulate mitotic progression. Nat Cell Biol 6(2): 138-145

Siu YT, Jin DY (2007) CREB - a real culprit in oncogenesis. FEBS J 274(13): 3224-3232

Song MS, Carracedo A, Salmena L, Song SJ, Egia A, Malumbres M, Pandolfi PP (2011) Nuclear PTEN regulates the APC-CDH1 tumorsuppressive complex in a phosphatase-independent manner. Cell 144(2): 187-199

Takahashi T, Yamasaki F, Sudo T, Itamochi H, Adachi S, Tamamori-Adachi M, Ueno NT (2005) Cyclin A-associated kinase activity is needed for paclitaxel sensitivity. Mol Cancer Ther 4(7): 1039-1046

Thyberg J (1984) The microtubular cytoskeleton and the initiation of DNA synthesis. Exp Cell Res 155(1): 1-8

Turnell AS, Stewart GS, Grand RJ, Rookes SM, Martin A, Yamano H, Elledge SJ, Gallimore PH (2005) The APC/C and CBP/p300 cooperate to regulate transcription and cell-cycle progression. Nature 438(7068): 690-695

Wang Q, Moyret-Lalle C, Couzon F, Surbiguet-Clippe C, Saurin JC, Lorca T, Navarro C, Puisieux A (2003) Alterations of anaphase-promoting complex genes in human colon cancer cells. Oncogene 22(10): 1486-1490

Wang TH, Wang HS, Soong YK (2000) Paclitaxel-induced cell death: where the cell cycle and apoptosis come together. Cancer 88(11): 2619-2628

Wilson BE, Mochon E, Boxer LM (1996) Induction of bcl-2 expression by phosphorylated CREB proteins during B-cell activation and rescue from apoptosis. Mol Cell Biol 16(10): 5546-5556

Yang E, Zha J, Jockel J, Boise LH, Thompson CB, Korsmeyer SJ (1995) Bad, a heterodimeric partner for $\mathrm{Bcl}-\mathrm{XL}$ and $\mathrm{Bcl}-2$, displaces Bax and promotes cell death. Cell 80(2): 285-291

Zachariae W, Schwab M, Nasmyth K, Seufert W (1998) Control of cyclin ubiquitination by CDK-regulated binding of Hct1 to the anaphase promoting complex. Science 282(5394): 1721-1724

Zha J, Harada H, Osipov K, Jockel J, Waksman G, Korsmeyer SJ (1997) BH3 domain of BAD is required for heterodimerization with BCL-XL and pro-apoptotic activity. J Biol Chem 272(39): 24101-24104

Zha J, Harada H, Yang E, Jockel J, Korsmeyer SJ (1996) Serine phosphorylation of death agonist BAD in response to survival factor results in binding to 14-3-3 not BCL-X(L). Cell 87(4): 619-628

Zhan Y, van de Water B, Wang Y, Stevens JL (1999) The roles of caspase-3 and bcl-2 in chemically-induced apoptosis but not necrosis of renal epithelial cells. Oncogene 18(47): 6505-6512

Zhang X, Odom DT, Koo SH, Conkright MD, Canettieri G, Best J, Chen H, Jenner R, Herbolsheimer E, Jacobsen E, Kadam S, Ecker JR, Emerson B, Hogenesch JB, Unterman T, Young RA, Montminy M (2005) Genomewide analysis of cAMP-response element binding protein occupancy, phosphorylation, and target gene activation in human tissues. Proc Natl Acad Sci USA 102(12): 4459-4464

This work is published under the standard license to publish agreement. After 12 months the work will become freely available and the license terms will switch to a Creative Commons Attribution-NonCommercial-Share Alike 3.0 Unported License. 